\title{
The impact of metoprolol on insulin sensitivity in the ICU
}

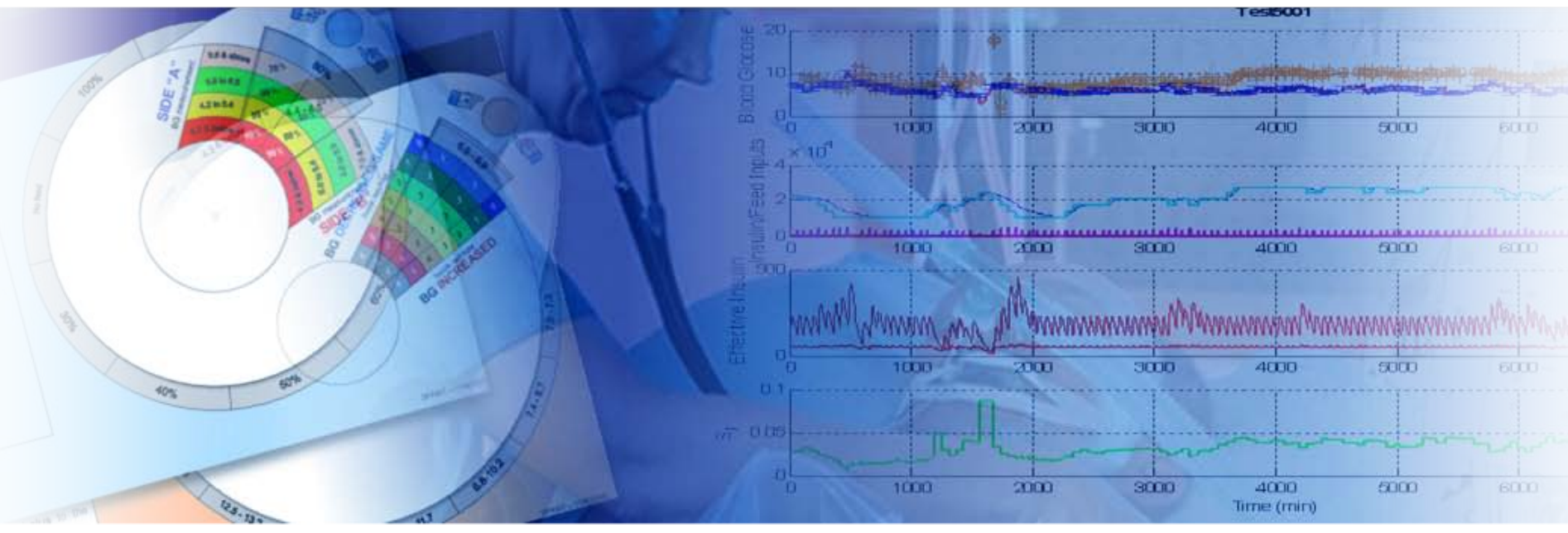

UNIVERSITY OTÄGO

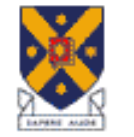

Tt Whane Wandingo e Otiag NEW ZEALAND
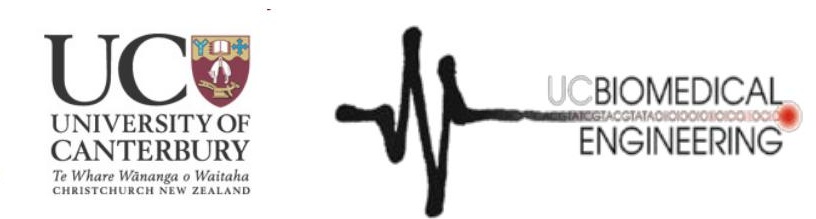

JG Chase et al

Dept of Mechanical Engineering Centre for Bio-Engineering University of Canterbury New Zealand 


\section{Introduction}

- Why do we care?

- Metoprolol is a a cardio-selective $\beta 1$-blocker regularly used in the Christchurch ICU

- In healthy subjects, metoprolol has been shown to reduce insulin sensitivity $14-27 \%$ when used to treat essential hypertension [Jacob 1996, Pollare 1989]

- Critically ill patients exhibit marked insulin resistant compared with healthy individuals

- If this effect extends to critically ill patients, we may be making TGC harder for ourselves

\section{Potentially worsening clinical outcomes}

\section{- Why Metoprolol?}

- A number of different $\beta$-blockers are used to treat various conditions in the ICU:

- Metoprolol (to reduce HR and contractility), Carvedilol (especially in congestive heart failure), Propranlol and Atenolol (to reduce HR and contractility)

- Metoprolol is one of the most frequently $\beta$-blockers used in the Christchurch ICU

- Metoprolol has been shown to reduce insulin sensitivity in healthy subjects

- This analysis methodology can be extended to other therapies eg. Corticosteroids 


\section{Background}

- Common characteristics of critically ill patients:

- Mechanical ventilation

- Sedation

- Enteral/parenteral nutrition

- Stress-response

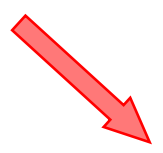

High levels of circulating counterregulatory hormones

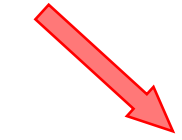

Insulin resistance
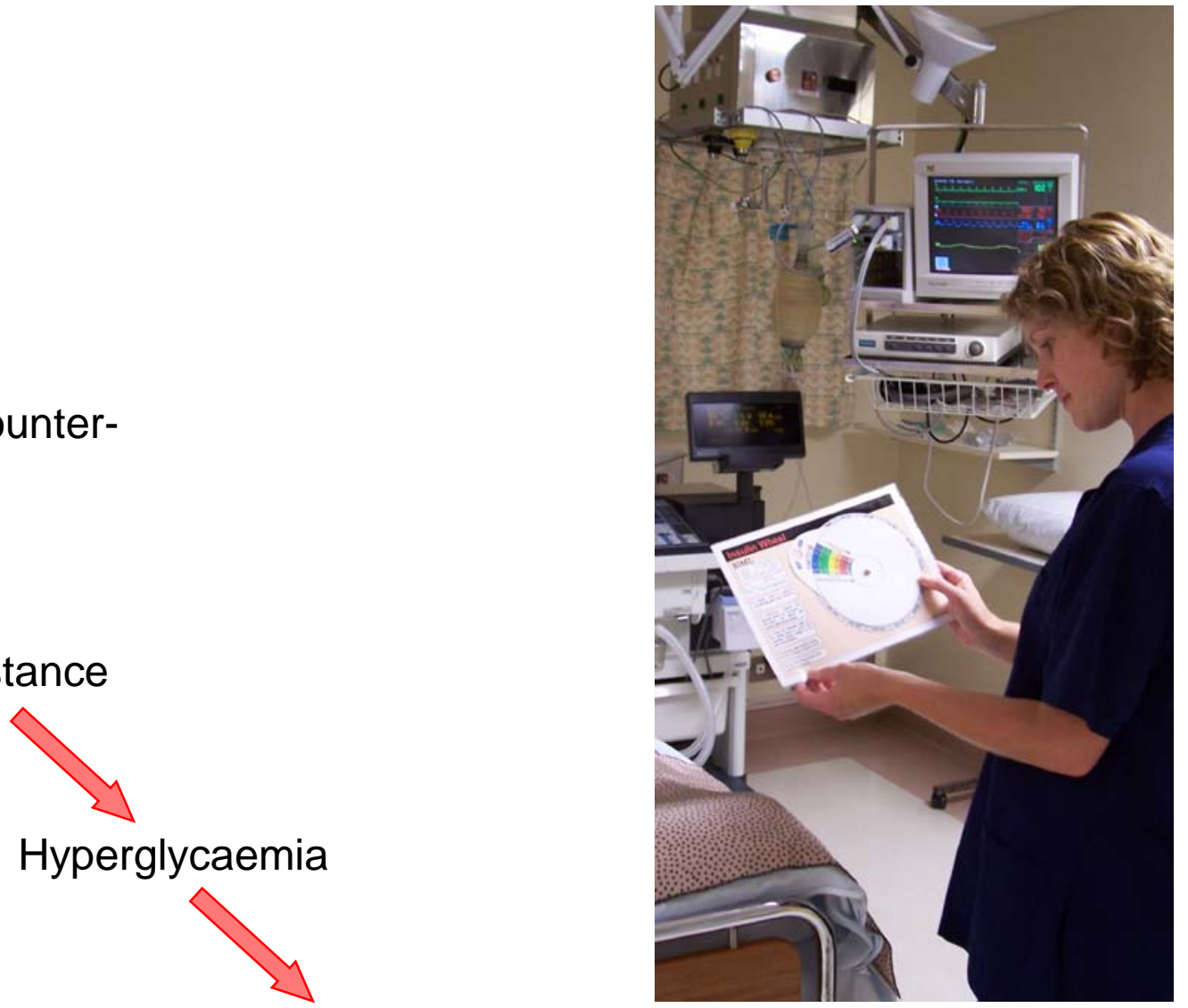

Increased morbidity and mortality 


\section{Background}

- What exactly is insulin sensitivity?

- A parameter quantifying insulin-mediated glucose uptake

- Insulin sensitivity (SI) $\propto$ Insulin resistance

- Low insulin sensitivity leads to hyperglycaemia

- ... and how can we measure it?

- The gold standard is the euglycaemic clamp

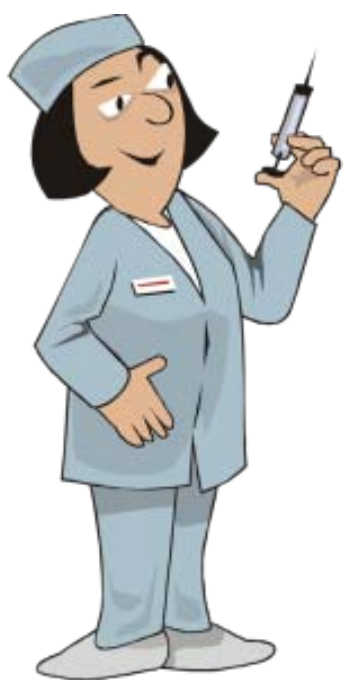

- A complicated procedure that can take 2+ hours requiring precise IV insulin and glucose and frequent blood sampling

- Results in an insulin sensitivity index (ISI)

Not easy to do with a critically ill patient! 
- A model-based approach

- Use model-based insulin sensitivity (SI)

- Clinically validated

- Correlates well with euglycaemic-clamp ISI $(r>0.90)$ [Lotz 2008]

- Provides a means to quantify $S I$ and its evolution over time in critically ill patients

- SI identified hourly for every patient

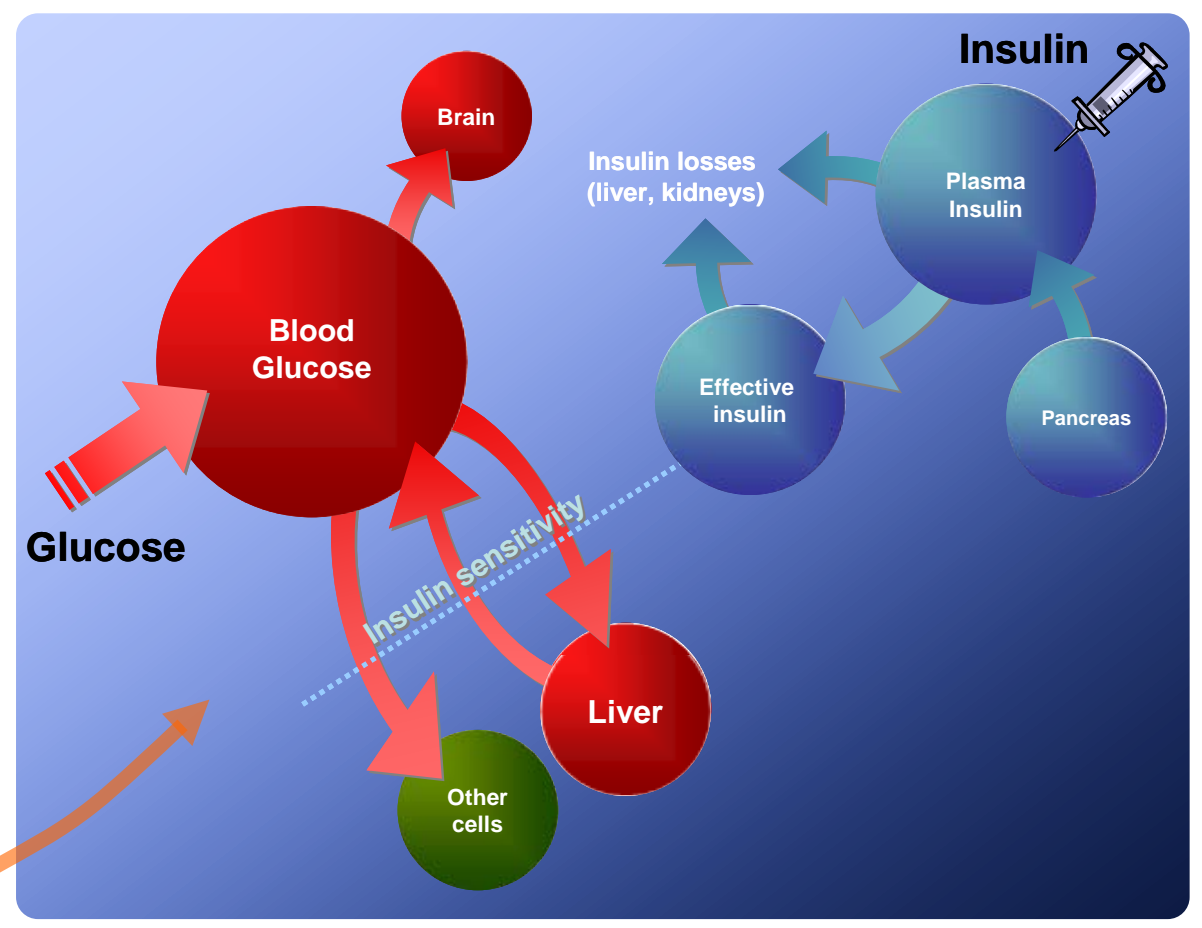

- BG system model

- ICING model [Lin 2010]

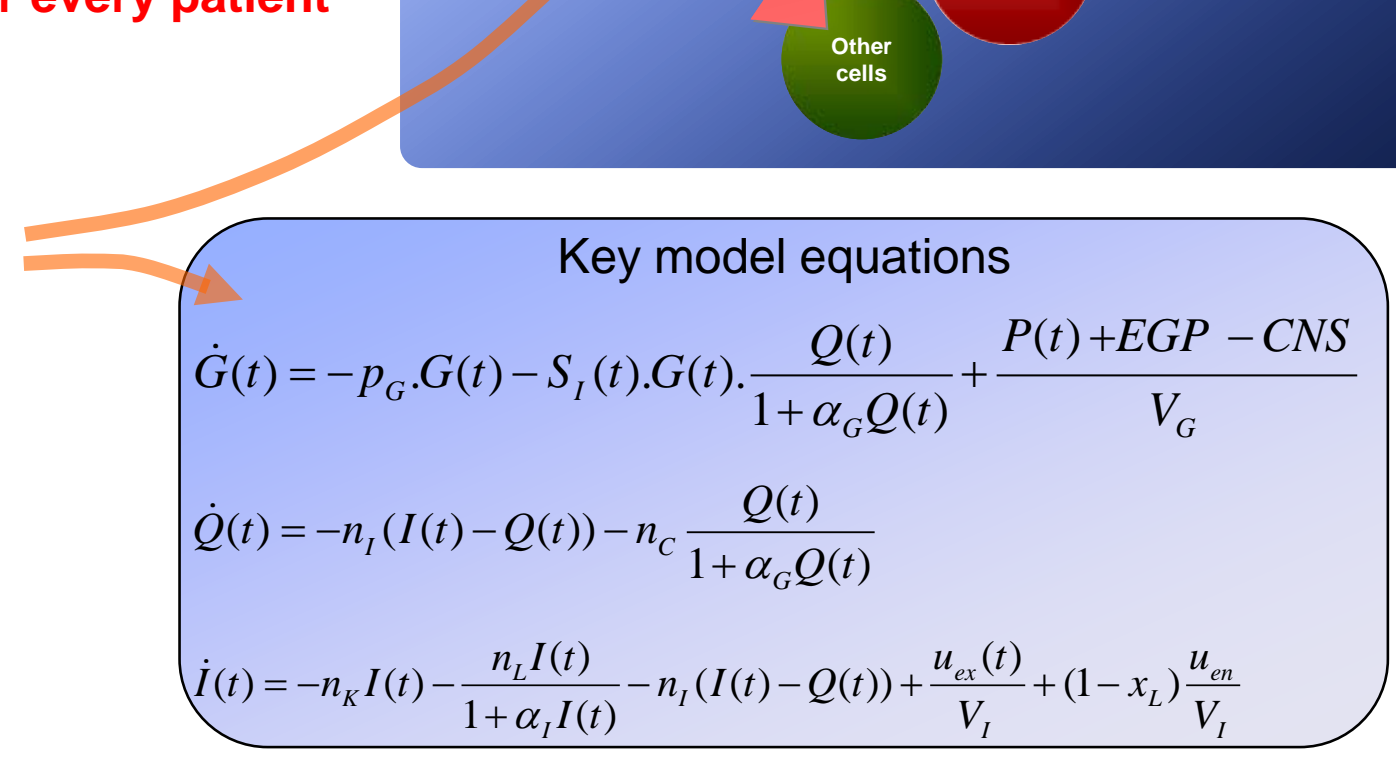




\section{Model}

\section{- Model-based SI}

- "Whole-body" insulin sensitivity

- Captures overall metabolic balance, including the relative net effect of :

- Altered endogenous glucose production

- Peripheral and hepatic insulin mediated glucose uptake

- Endogenous insulin secretion

- Has been used to guide model-based TGC in several studies

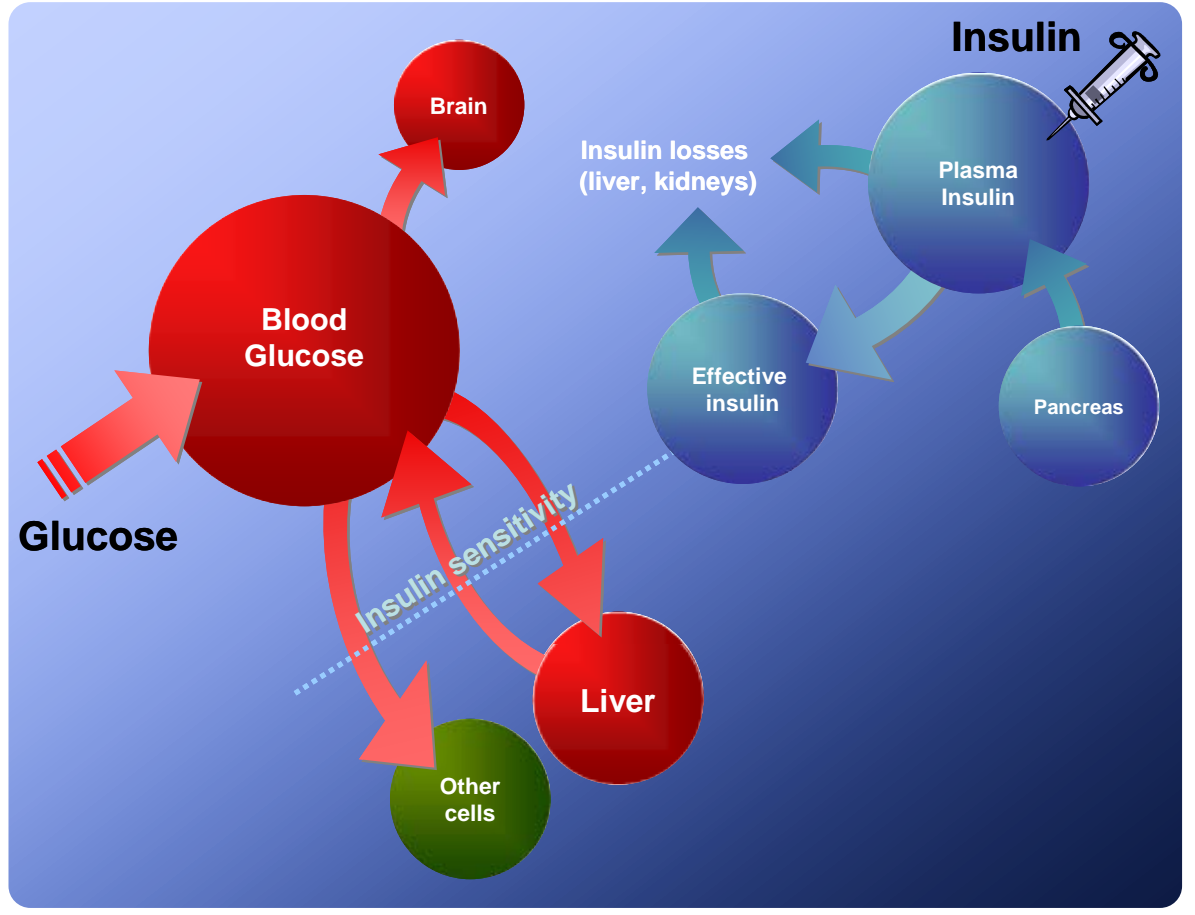




\section{Study cohorts}

\section{- Patients}

- Retrospective analysis of 34 patients from Christchurch hospital ICU

- 17 suitable patients receiving metoprolol were identified and 17 control patients were selected such that overall cohort statistics were matched

- All patients on the SPRINT glycaemic control protocol for $24 \mathrm{hrs}+$

- 3,369 and 4,126 S/ values were obtained for the control and metoprolol cohorts respectively.

- Patients were excluded if they were also receiving treatment with:

- Other $\beta$-blockers

- ACE inhibitors

- Corticosteroids

\begin{tabular}{|l|c|c|c|}
\hline & Control Cohort & $\begin{array}{c}\text { Metoprolol } \\
\text { Cohort }\end{array}$ & p-value \\
\hline $\mathbf{N}$ & 17 & 17 & \\
\hline Male/Female & $12 / 5$ & $13 / 4$ & $1.00^{* *}$ \\
\hline Op/Non-Op & $4 / 13$ & $4 / 13$ & $1.00^{* *}$ \\
\hline ICU Mortality & $35 \%$ & $24 \%$ & $0.71^{* *}$ \\
\hline Diabetic history & $3 / 14$ & $1 / 16$ & $0.60^{* *}$ \\
\hline Age (yrs) & $63[45-71]$ & $57[45-67]$ & $0.69^{*}$ \\
\hline APACHE II score & $19[16-27]$ & $20[16-26]$ & $0.70^{*}$ \\
\hline APACHE II ROD (\%) & $33.6[19.7-53.3]$ & $40.8[12.9-61.3]$ & $0.74 *$ \\
\hline $\begin{array}{l}\text { Patient median BG } \\
\text { (mmol/L) }\end{array}$ & $5.7[5.0-6.4]$ & $5.8[5.1-6.5]$ & $0.11^{*}$ \\
\hline $\begin{array}{l}\text { ICU Length of stay } \\
\text { (hrs) }\end{array}$ & $302[139-512]$ & $360[178-655]$ & $0.63^{*}$ \\
\hline $\begin{array}{l}\text { Patient time on } \\
\text { SPRINT (hrs) }\end{array}$ & $141[88-293]$ & $178[73-339]$ & $0.45^{*}$ \\
\hline $\begin{array}{l}\text { Total time on } \\
\text { SPRINT (hrs) }\end{array}$ & 3369 & 4126 & \\
\hline $\begin{array}{l}\text { Daily dose of } \\
\text { metoprolol (mg/d) }\end{array}$ & 0 & $100[50-200]$ & \\
\hline $\begin{array}{l}\text { Total time on } \\
\text { metoprolol (hrs) }\end{array}$ & 0 & 3079 & \\
\hline
\end{tabular}

\begin{tabular}{|c|c|c|c|}
\hline \multicolumn{2}{|c|}{ Diagnostic category } & $\begin{array}{c}\text { Control cohort } \\
\text { patients }\end{array}$ & $\begin{array}{c}\text { Metoprolol } \\
\text { cohort patients }\end{array}$ \\
\hline \multirow{6}{*}{ 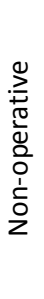 } & Cardio & 4 & 5 \\
\hline & Respiratory & 2 & 2 \\
\hline & Gastro & 2 & 1 \\
\hline & Sepsis & 0 & 1 \\
\hline & Trauma & 4 & 4 \\
\hline & Other (Renal etc) & 1 & 0 \\
\hline \multirow{3}{*}{ 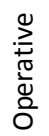 } & Cardio & 2 & 1 \\
\hline & Gastro & 1 & 3 \\
\hline & Trauma & 1 & 0 \\
\hline
\end{tabular}

${ }^{*} p$-values calculated with Mann-Whitney U-test.

**p-values calculated with two-sided Fishers exact test. 


\section{Study details}

- Metoprolol

- For this study, SI was considered to be affected by the drug for 12 hours following the last dose

- 3079 SI values affected by metoprolol

- Median daily dosage for this cohort: 100 [50-200] mg/d

- Studies have shown duration of action for heart-rate and bloodpressure effects of 12-24 hours for oral doses of 100200 mg/day [Åblad 1975, Freestone 1982, Johansson 1980..]

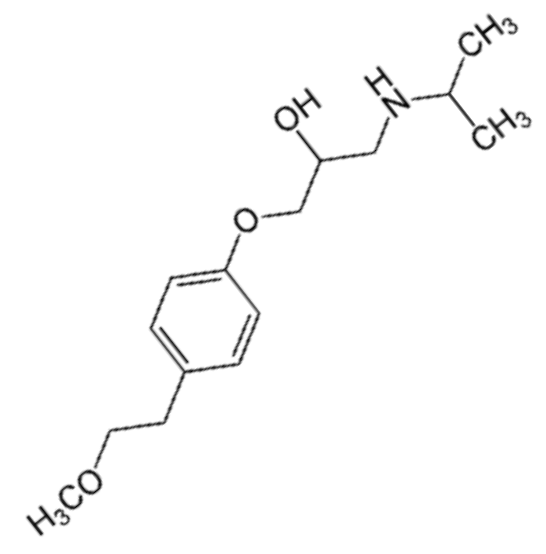

- The variable and prolonged effect of metoprolol made comparison of the insulin sensitivity within the metoprolol cohort between periods on and off the treatment unfeasible as there are few hours of data that could confidently be considered unaffected by the drug

\section{- SPRINT}

- Tight glycaemic control (TGC) protocol used in Christchurch hospital ICU since August 2005

- A simple, lookup-table system derived from a model-based controller

- Titrates insulin doses and nutrition rates to patient-specific

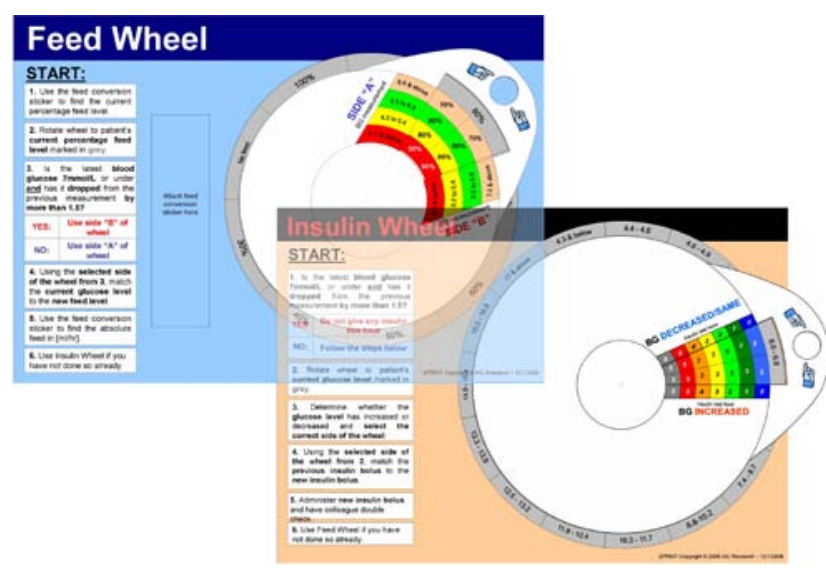
insulin sensitivity 
- Quantifying differences in SI between cohorts

- Typical distributions of SI are asymmetric and skewed

- Non-parametric statistics are used (median, interquartile range)

- Cumulative distribution functions (CDFs)

- Cohort comparisons

- Percentile-patient comparisons

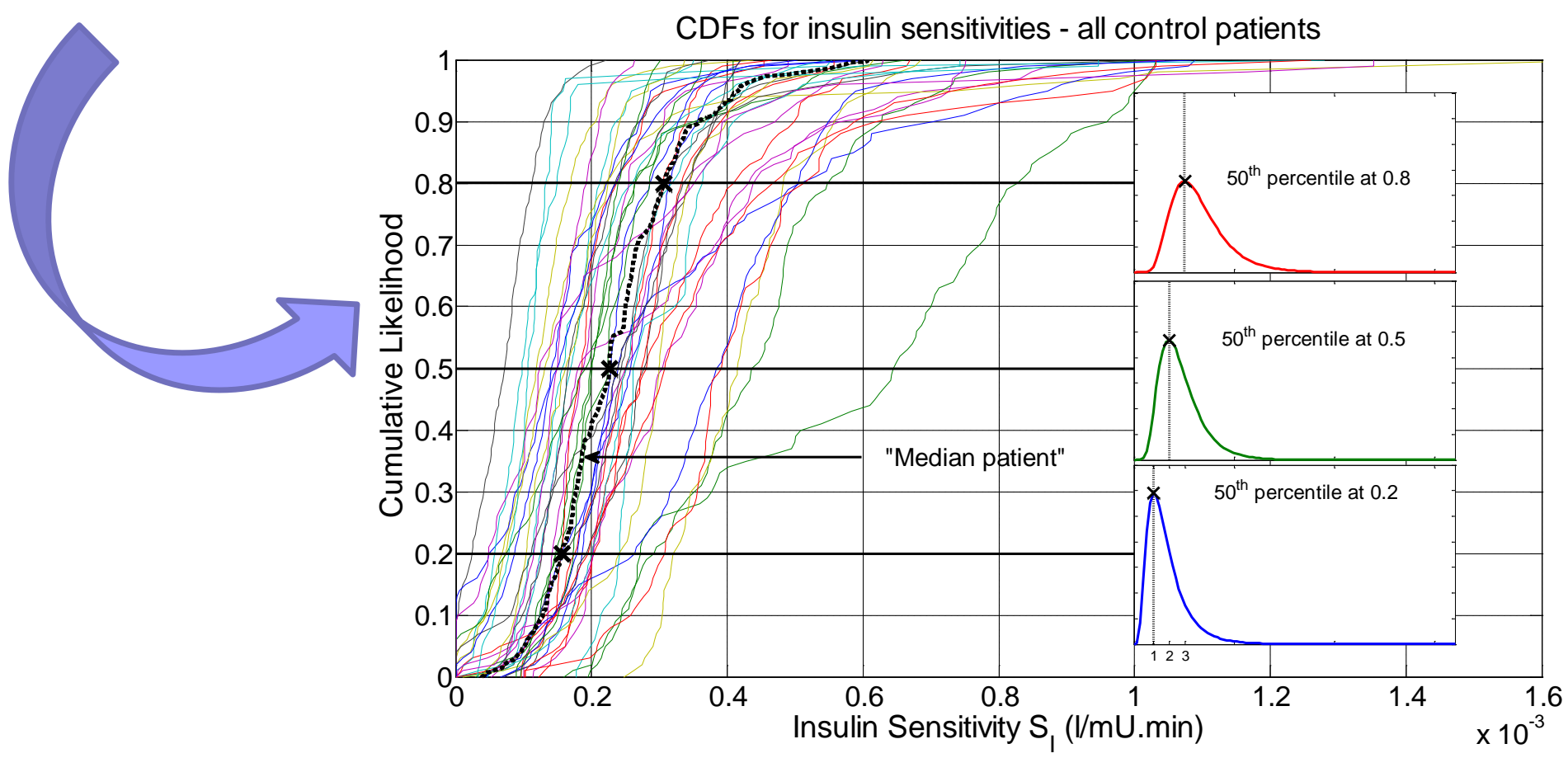




\section{- Cohort Analysis}

- Metoprolol is associated with a $\mathbf{9 . 7 \%}$ reduction in SI at the median compared to controls

- Control median SI: $4.04 \times 10^{-4}$ L/mU.min

- Metoprolol median SI: 3.65×10-4 L/mU.min

- $\quad$ P 0.05 (Mann-Whitney U-test)

- Clear separation between

Cohort comparison of insulin sensitivity cohorts the $10^{\text {th }}$ and $90^{\text {th }}$ percentiles.

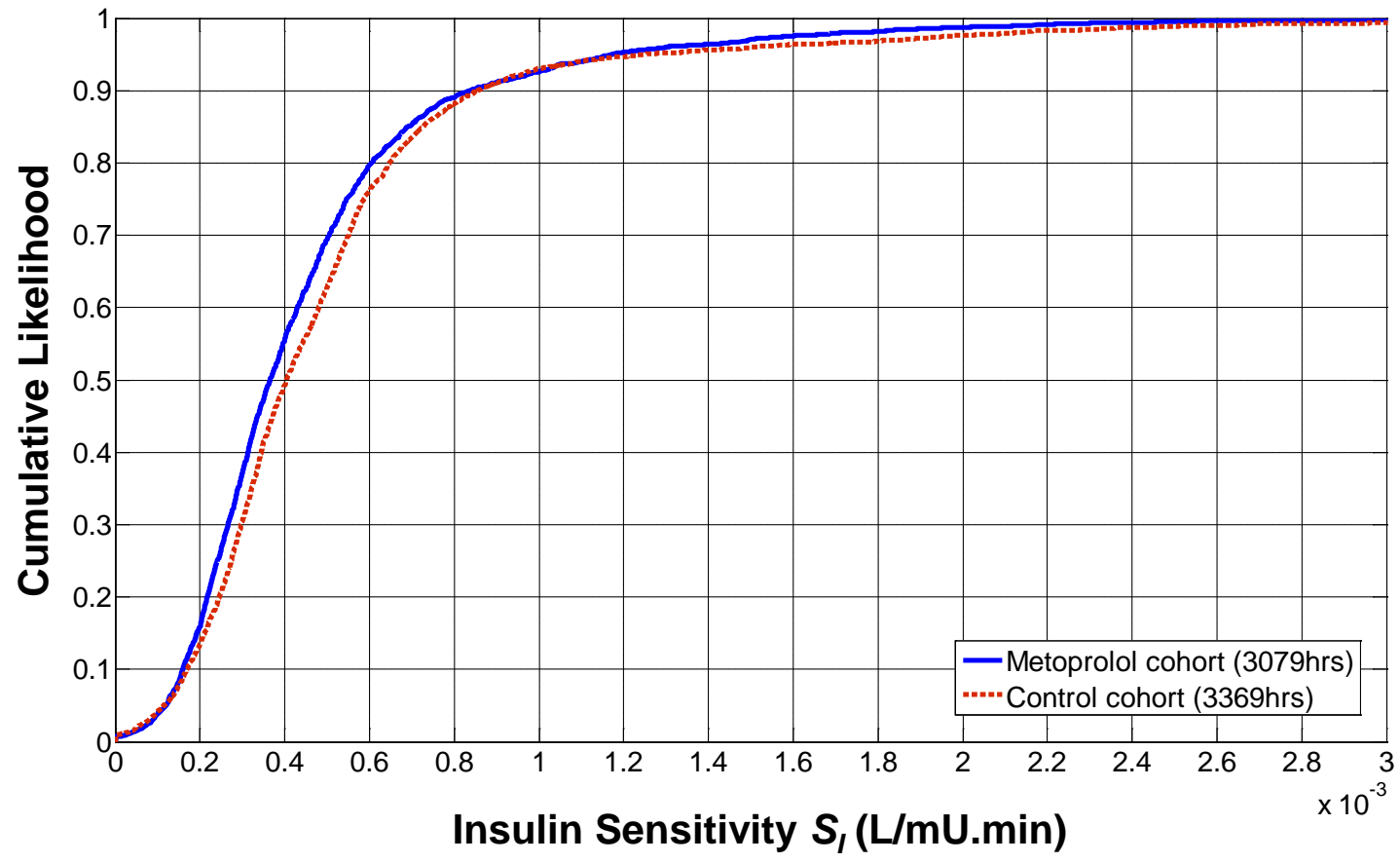




\section{Results}

\section{- Percentile-Patient Analysis}

- No statistically significant differences in $S I$ distributions for $25^{\text {th }}-, 50^{\text {th }}$ - and $75^{\text {th }}$-percentile patients

- Differences at the median in the range 0.5-3.6\%

- $\quad \mathbf{P}>\mathbf{0 . 2}$ for these three comparisons (Mann-Whitney U-test)

- Small cohorts means outlying percentile patients (e.g. $5^{\text {th }}$ or $\left.95^{\text {th }}\right)$ are heavily influenced by individual outlying patients in each cohort.

Percentile-patient comparison of insulin sensitivity cohorts

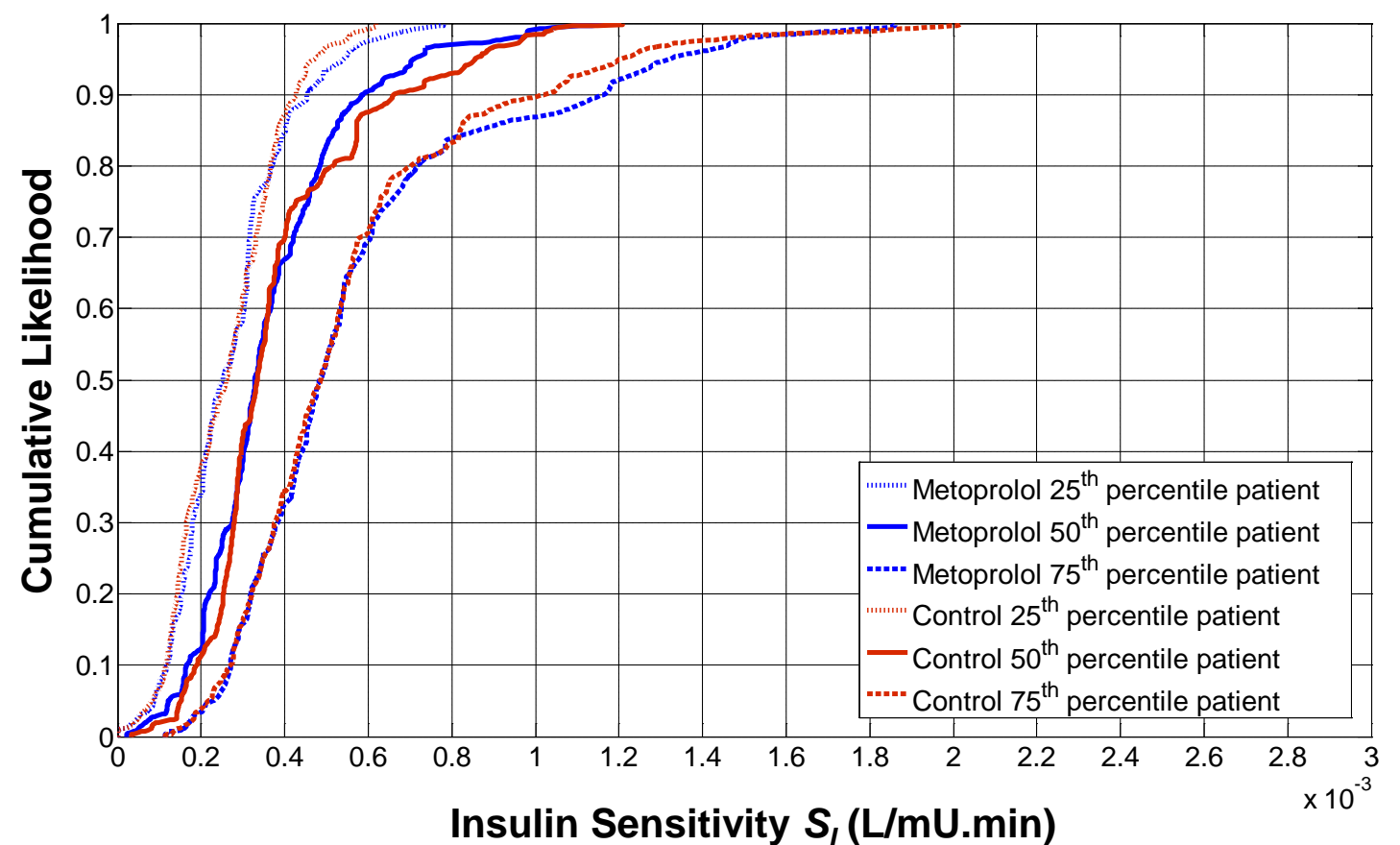




\section{Summary of Results}

- Metoprolol and healthy people

- $14-27 \%$ reduction in insulin sensitivity for non-diabetic subjects

- Doses of 100-200 mg/d [Jacob 1996, Pollare 1989]

- $2 \%$ reduction in insulin sensitivity for NIDDM subjects (statistically non-significant)

- Doses of 50-200 mg/d additional to existing ACE-inhibitor or ARB hypertension treatment regime (can increase $\mathrm{SI}$ ) may have affected results for this particular study. [Falkner 2008]

- Metoprolol and critically ill patients

- $\quad 0.5-10 \%$ reduction in SI associated with Metoprolol isn't clinically significant

- Median daily dosage for this cohort: 100 [50-200] mg/d

- Metoprolol-mediated reduction of Sp is limited in critically ill patients

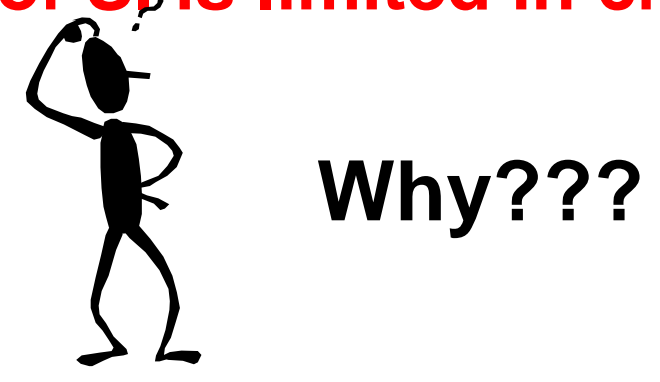




\section{Discussion}

\section{- Why do we see limited reductions in SI with critically ill patients?}

- The mechanisms by which $\beta$-antagonist treatment ( $\beta$-blockade) modifies insulin sensitivity are not yet understood

- Haemodynamic explanations have been proposed [Jacob 1996, Pollare 1989]

- Reduced heart rate and contractility $\rightarrow$ reduced blood flow to the skeletal muscles.

- Skeletal muscles are prime target tissues for insulin-mediated glucose disposal.

- Receptor downregulation in response to reduced insulin clearance associated with $\beta$-blockade has also been suggested [Jacob 996]

- Insulin clearance $\downarrow \rightarrow$ plasma insulin concentration $\uparrow \rightarrow$ receptor downregulation $\rightarrow$ insulin mediated glucose uptake $\downarrow$

- The critical condition of the patients in this study may moderate the physiological impact of these proposed mechanisms of action

- Critically ill patients already have significant peripheral insulin resistance and may be less likely to show further large reductions caused by reduced blood flow or receptor downregulation compared to healthy subjects

- Cohort/patient differences (a limitation)

- Individual patients are not matched between cohorts.

- $\quad$ Very hard to find patients on beta blockers and not on glucocorticoids (similar effects) or ACE inhibitors (given in conjunction to reduce hypertension but have opposite effects) 
- SI reductions associated with metoprolol are considerably less in critically ill patients compared with healthy subjects

- $0.5-10 \%$ compared to $14-27 \%$

- Because critically ill patients are already significantly insulin resistant, the physiological impact of the suggested mechanisms of action may be moderated

- Therefore, the use of metoprolol is likely to have minimal impact on glycaemic control in the ICU setting

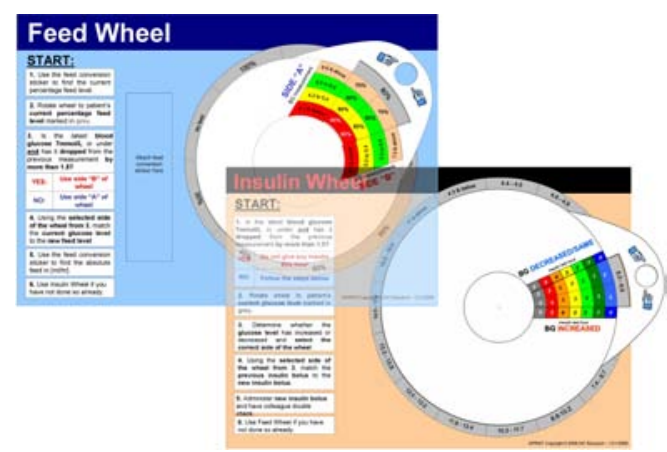




\section{Don't Forget ...}

\section{(8)}

8th IFAC SYPOSIUMON ON BIOLOGICAL AND MEDICAL SYSTEMS

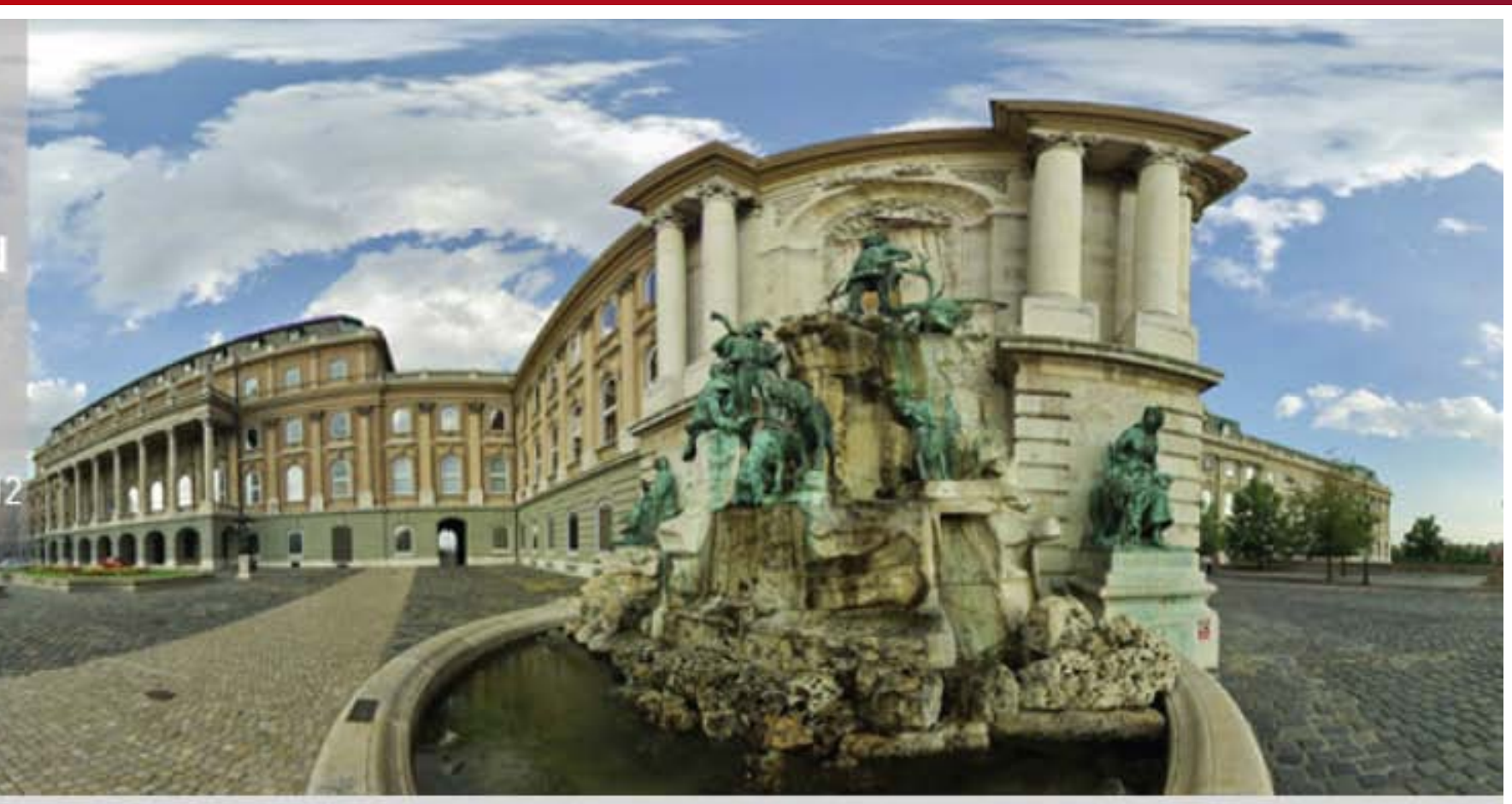

8th IFAC Symposium on Biological and Medical Systems

Budapest, Hungary / 29-31 August, 2012

\section{bms.iit.bme.hu}


